Arch. Tierz., Dummerstorf 44 (2001) 2, 167-179 Aus dem Institut fur Tierzuchtwissenschuft der Universitat Bonn', dem Institut fir Tierzucht und Haustiergenetik der
Universitat Gottingen ${ }^{2}$, der Zuchtungszentrale Deutsches Hybridschwein in Laneburg' und dem Schweinezuchter-
verband Nord-West

ERNST THOLEN', HORST BRANDT ${ }^{2}$, HUBERT HENNE ${ }^{3}$, FRANZ-JOSEF STORK ${ }^{4}$ und KARL SCHELLANDER'

\title{
Genetische Fundierung von AutoFOM-Merkmalen
}

\author{
Herrn Professor Dr. Erhard Kallweit zum 65. Geburtstag gewidmet
}

\begin{abstract}
Summary
Title of the paper: Genetic foundation of AutoFOM-traits

Since the beginning of the year 2000 the payment system of pig carcasses in some slaughter houses in northwest Germany is based on the recordings of the fully automated carcass classification system AutoFOM. Besides legal information 'estimated lean meat percentage' relative exact details of the weights of important valuable cuts are available. Genetic parameters of the AutoFOM lean carcass cut information, ham-, chop-, shoulder-and belly weights as well as lean meat percentage of the belly cut were estimated. Using the field data of the pig breeding organisations BHZP $(n=2224)$ and SNW $(n=3601)$ the estimated heritabilties of these carcass cuts vary between 0.13 and 0.27 (BHZP) and 0.13 and 0.31 (SNW), respectively. The estimated heritabilities of the AutoFOM carcass traits of station-tested pigs were more pronounced for the dam lines German Landrace and Large White $(n=1693)$ and Piétrain AI-boars mated to FI sows $(n=1053)$. The estimates ranged from 0.27 to 0.59 (dam lines) and 0.05 to 0.40 ( $\mathrm{Pi}$ Al-boars), respectively. Using the records of station tested purebred Piétrain pigs $(n=1693)$ the heritabilities of the AutoFOM carcass cuts did not exceed 0.10 . The genetic correlation between the different AutoFOM carcass traits exceed the absolute value of 0.8 , In a similar way, the genetic correlation between the 'estimated lean meat percentage' used in the test station and the AutoFOM carcass traits were close to unity. We concluded that AutoFOM traits of slaughter pigs provide a useful information for the selection of AI-boars. Moreover, with regard to the station testing of dam lines AutoFOM carcass informations derived from AutoFOM are a useful supplementation. Because of the low heritability, only a marginal additional benefit could be expected for the genetic ranking of Pi-boars using AutoFOM carcass cut records of purebred station tested progenies.
\end{abstract}

Key Words: pig, AutoFOM, genetic foundation, field and station test

\section{Zusammenfassung}

Die vollautomatische Klassifizierung von Schlachtkðrpern mit Hilfe des AutoFOM-Gerates ist seit Beginn des Jahres 2000 in einigen Schlachtunternehmen des nordwestdeutschen Raumes Grundlage des. Bezahlungssystems von Schlachtschweinen. Neben dem gesetzlich vorgeschriebenen Muskelfleischanteil liefert das AutoFOM-Gerăt vergleichsweise exakte Schătzwerte für die Ausprägung der wertbestimmenden Teilstucke. Mit Hilfe von im Feld und in der Station geproften Schweinen erfolgte eine Schatzung (REML, Tiermodell) der genetischen Parameter der AutoFOM-Merkmale Schinken-, Schulter-, Lachs-, und Bauchgewicht sowie dem Bauchfleischanteil. Die Heritabilitaten der AutoFOM-Schlachtkðrpermerkmale, die mit Hilfe der Produktionsdatensätze der Unternehmen BHZP $(\mathrm{N}=2224)$ und SNW $(\mathrm{N}=3601)$ geschatzt wurden, reichen von 0,13 bis 0,27 ( $\mathrm{BHZP})$ bzW. 0,13 bis 0,31 (SNW). Auf einem etwas hơheren Niveau lagen die geschátzten Heritabilităten der AutoFOMMerkmale, die mit Hilfe der Stationsdatensä́tze der Herkunft Piétrain " FI $(\mathrm{N}=1053)$ sowie den Mutterlinien Deutsches Edelschwein und Deutsche Landrasse $(\mathrm{N}=1228)$ ermitteltet wurden. Fur die AutoFOM-Schlachtkörpermerkmale wurden bei den Mutterlinien mittlere $\mathrm{h}^{2}$-Werte im Bereich von 0,27 bis 0,59 bzw. bei den $\mathrm{Pi}-\mathrm{Be}$ samungsebern von 0,05 bis 0,40 geschätzt, Bei den in Reinzucht gepruften Pi-Schweinen $(\mathrm{N}=1693)$ konnten mit 0,02 bis 0,10 deutlich niedrigere Werte festgestellt werden. Sowohl bei der Analyse der Produktionsdatensătze als auch der Stationsdaten lagen die geschatzzen Korrelationen zwischen den AutoFOM-Merkmalen und zwischen den stationär erfassten Merkmalen Fleischanteil nach „Bonner Formel“ und den AutoFOM-Merkmalen im 
Absolutbetrag aber 0,8. Unsere Ergebnisse zeigen, dass AutoFOM Merkmale von Produktionsschweinen zur Rangierung von Besamungsebern züchterisch genutzt werden kø̋nen. Daruber hinaus stellen AutoFOM-Merkmale bei Mutterlinien eine sinnvolle Ergänzung der aufwendigen Stationsprifung dar, wăhrend der zuchterische Wert fur Pi-Reinzuchttiere auf Grund der niedrigen Heritabilităten als gering einzuschätzen ist.

Schlusselworter: Schwein, AutoFOM, genetische Fundierung, Feld- und Stationsprufung

\section{Einleitung}

Die vollautomatische Klassifizierung von Schlachtkörpern mit Hilfe des AutoFOMGerates (SFK, Company, DK) ist seit Beginn des Jahres 2000 in einigen nordwestdeutschen Schlachtunternehmen Grundlage des Bezahlungssystems von Schlachtschweinen. Die wesentlichen Vorteile dieses Verfahrens gegenüber dem weityerbreitenden FOM-Sonden Klassifizierungsgerăt liegen in der höheren Schätzgenauigkeit, dem fehlenden Bedienereinfluss sowie in den hohen, erreichbaren Schlachtbandgeschwindigkeiten. Zusätzlich zum gesetzlich vorgeschriebenen Muskelfleischanteil liefert das AutoFOM-Gerät Schätzwerte für die Gewichte der wertbestimmenden Teilstilcke Bauch, Kotelett, Schinken und Schulter, die eine genauere Aussage über den tatsăchlichen Handelswert von Schlachtkörpern ermöglichen.

Die Eignung des AutoFOM-Meßgerätes zur Abschätzung des wahren Fleischanteils und damit des Handelswertes der Schlachtkörper wurden in den Untersuchungen von BRANSCHEID et al. (1997, 1999) und BRONDUM et al. (1998) bestätigt. Die AutoFOM-Merkmale sind Grundlage des Bezahlungssystems einiger wichtiger nordwestdeutscher Schlachtunternehmen (BEUCK, 1999); hieraus ergibt sich deren ökonomische Relevanz. Ungeklärt hingegen sind die Fragen: a) Welche genetische Fundierung besitzen die AutoFOM-Merkmale? b) Welche Beziehungen bestehen zwischen den AutoFOM-Merkmalen und den Schlachtkörpermerkmalen, die im Rahmen der stationären Leistungsprüfungen erhoben werden? Die vorliegende Untersuchung soll einen Beitrag zu beiden Fragestellungen liefern.

\section{Material und Methoden}

Die Schätzung der genetischen Parameter erfolgt mit Hilfe von Daten, die im Rahmen der Top-Genetik Prüfung von Besamungsebern a) unter Produktionsbedingungen und b) unter den Bedingungen der stationären Geschwister-/Nachkommen Prüfung beim Schwein (ALZ, 1999) erfasst wurden. Die Produktionsdaten stammen aus 5 Testbetrieben des Schweinezuchtunternehmens BHZP mit den Herkünften Endstufeneber Linie 65, 66 und $67^{*} \mathrm{db}$-Sau (Datensatz BHZP) und aus 6 Betrieben der Schweinezilchterverbandes Nord-West mit der Herkunf Pi * Westhybrid Sau (Datensatz SNW) (Tab. 1).

Die stationären Prüfergebnisse wurden in den Leistungsprüfanstalten Haus Düsse und Frankenforst erhoben. Die Prüfung erfolgte mit Reinzucht (Deutsche Landrasse, Deutsches Edelschwein, Piétrain) bzw. Kreuzungstieren DE*DL, DL*DE und Pi*F1 der beiden nordrhein-westfälischen Herdbuchzuchtverbände Schweinezüchterverband Nord-West und Landesverband Rheinischer Schweinezïchter (Datensatz Station). Der Erfassungszeitraum fừ die Datensätze BHZP, SNW und Station reichen vom Mai 1999 bis Nov. 1999, Mai 1999 bis Dez. 1999 bzw. Juli 1998 bis Feb. 2000. 
Tabelle 1

Struktur der analysierten Datensătze (Structure of the analysed data sets)

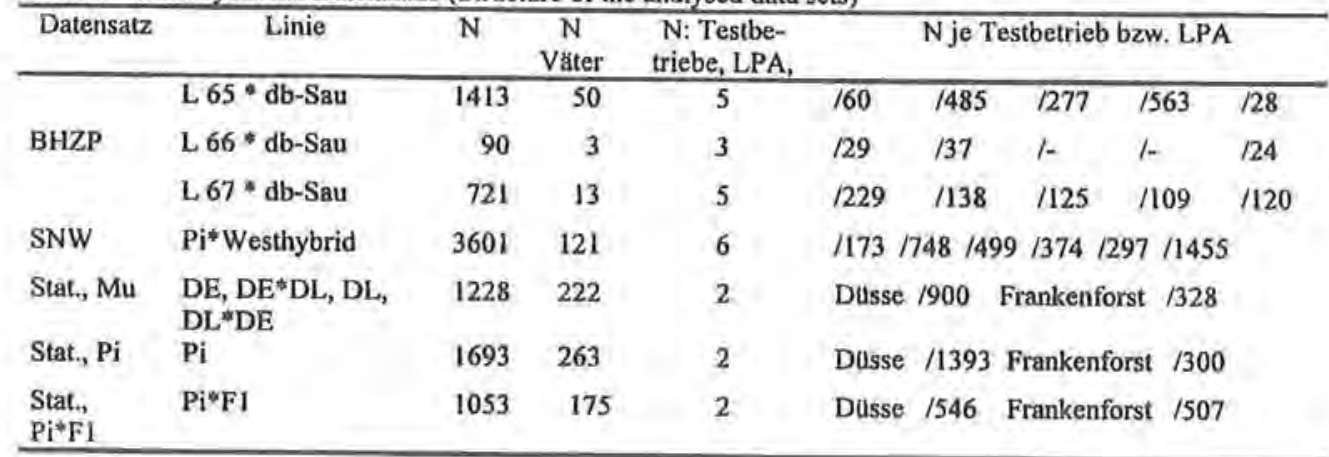

Die Mittelwerte und Standardabweichungen der Produktionsdatensätze (Tab. 2) zeigen nur unwesentliche Unterschiede zwischen den beiden beteiligten Zuchtorganisationen. Die unterschiedlichen Produktionsbedingungen in den beteiligten Testbetrieben lassen eine weiterführende Interpretation der Mittelwertsdifferenzen zwischen beiden Datensätzen nicht zu.

Tabelle 2

Mittelwert und Standardabweichung, Produktionsdaten (Means and standard deviations, data from fatting herds)

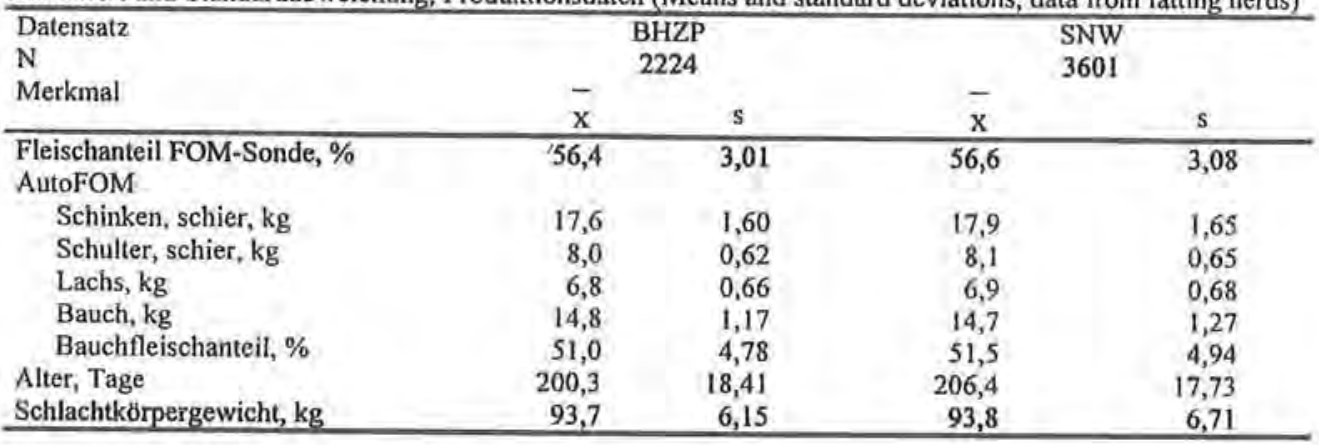

Die Daten der Stationsprüfung (Tab, 3) zeigen die bekannten Unterschiede zwischen den gepriften Herkunften. Die Rasse Pi weist eine deutliche Überlegenheit in allen Schlachtkörpermerkmalen auf, während die Tiere der Mutterlinien aufgrund der höheren Futteraufnahme deutliche Vorteile im Merkmal Tägliche Zunahme im Mastabschnitt 30-105 kg Lebendgewicht besitzen. Die Pi*Fl-Endprodukte nehmen in allen Merkmalen eine Mittelstellung ein. Die gewichtsmäßig geschätzten AutoFOM-Merkmale dieser Endprodukte liegen im Vergleich zu den Produktionsdatensätzen auf einem niedrigeren Niveau. Das im Rahmen der Stationsprüfung vorgegebene Schlachtgewicht von $85 \mathrm{~kg}$ ist hierfür als Erklärung heranzuziehen.

Die zur Zeit in den Herdbuchverbänden stattfindende Eliminierung des positiven Stressallels P des MHS (Malignen Hyperthermie Syndrom) Genortes hat dazu geführt, dass sowohl bei den Pi-Reinzuchttieren als auch bei den $\mathrm{Pi}^{*} \mathrm{~F} 1$-Endprodukten bezüglich des MHS-Status unterschiedliche Genotypen stationär geprüft wurden. Tabelle 4 beinhaltet eine Auflistung des je nach MHS-Status erreichten Leistungsniveaus. Hier- 
bei zeigt sich im Merkmal Fleischanteil nach Bonner Formel eine Überlegenheit der homozygot stressanfälligen Pi-Tieren gegenüber den NN- und NP-MHS-Genotypen von 1,88 bzw. 1,17 \%. Bezogen auf eine Standardabweichung sind Unterschiede zwischen den MHS-Genotypen bei den übrigen AutoFOM-Merkmalen in ähnlicher Grössenordnung festzustellen.

Tabelle 3

Mittelwert und Standardabweichung, Stationsdaten (Means and standard deviations, data from station test)

\begin{tabular}{|c|c|c|c|c|c|c|c|}
\hline \multirow{2}{*}{\multicolumn{2}{|c|}{$\begin{array}{l}\text { Datensatz } \\
\mathrm{N} \\
\text { Merkmal }\end{array}$}} & \multicolumn{2}{|c|}{$\begin{array}{l}\text { PI } \\
1693\end{array}$} & \multicolumn{2}{|c|}{$\begin{array}{c}\mathrm{Mu} \\
1228\end{array}$} & \multicolumn{2}{|c|}{$\begin{array}{l}\mathrm{Pi} \cdot \mathrm{F} 1 \\
1053\end{array}$} \\
\hline & & $\mathrm{x}$ & s & $\mathbf{x}$ & s & $\mathrm{x}$ & s \\
\hline Fleischanteil, FOM-Sonde & & 61,1 & 2,12 & 51,5 & 3,44 & 57,9 & 2,36 \\
\hline \multicolumn{8}{|l|}{ AutoFOM } \\
\hline Schinken, schier, kg & & 17,7 & 1,12 & 14,5 & 1,47 & 16,7 & 1,26 \\
\hline Schulter, schier, kg & & 7,7 & 0,39 & 6,9 & 0,50 & 7,5 & 0,37 \\
\hline Lachs, kg & & 6,8 & 0,50 & 5,7 & 0,52 & 6,4 & 0,47 \\
\hline Bauch, kg & & 13,0 & 0,65 & 13,9 & 0,80 & 13,2 & 0,61 \\
\hline Bauchfleischanteil, \% & & 56,6 & 0,32 & 43,9 & 0,40 & 53,8 & 0,31 \\
\hline \multicolumn{8}{|l|}{ LPA } \\
\hline Zunahme & im & 789,2 & 77,91 & 938,3 & 89,92 & 843,1 & 81,21 \\
\hline \multicolumn{8}{|l|}{ Prufabschnitt, g/Tag } \\
\hline Schlachtkßrpergewicht, kg & & 85,1 & 2,60 & 85,4 & 2,73 & 85,2 & 2,69 \\
\hline Fleischflache, $\mathrm{cm}^{2}$ & & 59,2 & 5,23 & 42,3 & 4,19 & 53,1 & 4,73 \\
\hline Fettflache, $\mathrm{cm}^{2}$ & & 12,3 & 2,19 & 21,0 & 3,24 & 15,5 & 2,40 \\
\hline Ruckenspeckdicke, cm & & 1,9 & 0,26 & 2,7 & 0,35 & 2,2 & 0,30 \\
\hline Seitenspeckdicke, cm & & 2,0 & 0,63 & 3,7 & 0,84 & 2,4 & 0,73 \\
\hline Speckmaß a. RMF, cm & & 0,8 & 0,22 & 1,8 & 0,37 & 1,1 & 0,24 \\
\hline Lange, $\mathrm{cm}$ & & 96,7 & 2,60 & 101,6 & 2,50 & 99,7 & 2,51 \\
\hline Fleischanteil, BF, \% & & 63,9 & $\mathrm{I}, 98$ & 55,2 & 2,12 & 60,9 & 1,93 \\
\hline $\begin{array}{l}\text { Leitrhigkeit, Kotelett, } \\
24 \text { h p.m., mS/ }\end{array}$ & & 5,9 & 2,92 & 2,6 & 0.82 & 3,4 & 1,31 \\
\hline
\end{tabular}

Tabelle 4

Mittelwerte und Standardabweichungen der stationar erfassten Merkmale, getrennt nach Herkunft und MHSStatus (Means and standard deviations of traits from station test, divided into breed and MHS-status classes)

\begin{tabular}{|c|c|c|c|c|c|c|c|c|c|c|}
\hline \multirow{4}{*}{$\begin{array}{l}\text { Datensatz } \\
\text { MHS Starus } \\
\mathrm{N} \\
\text { Merkmal }\end{array}$} & \multicolumn{6}{|c|}{$\mathrm{Pi}$} & \multicolumn{4}{|c|}{$\mathrm{Pi}^{*} \mathrm{~F} 1$} \\
\hline & \multirow{2}{*}{\multicolumn{2}{|c|}{$\begin{array}{l}\text { NN } \\
78\end{array}$}} & \multirow{2}{*}{\multicolumn{2}{|c|}{$\begin{array}{l}\text { NP } \\
569\end{array}$}} & \multirow{2}{*}{\multicolumn{2}{|c|}{$\begin{array}{l}\text { PP } \\
890\end{array}$}} & \multirow{2}{*}{\multicolumn{2}{|c|}{$\begin{array}{c}\mathrm{NP}^{*} \mathrm{NN} \\
338\end{array}$}} & \multirow{2}{*}{\multicolumn{2}{|c|}{$\begin{array}{l}\text { PP } \times N N \\
715\end{array}$}} \\
\hline & & & & & & & & & & \\
\hline & $\bar{x}$ & $\mathbf{s}$ & $\bar{x}$ & $\mathbf{s}$ & $\bar{x}$ & s & $\bar{x}$ & $\mathrm{~s}$ & $\bar{x}$ & $s$ \\
\hline Fieischanteil, FOM & 59,2 & 2,31 & 60,2 & 2,20 & 61,6 & 2,42 & 57,9 & 2,43 & 57,9 & 2,74 \\
\hline AutoFOM & & & & & & & & & & \\
\hline Schinken, schier, kg & 17,0 & 1,06 & 17,5 & 1,05 & 17,9 & 1,12 & 16,7 & 1.08 & 16,7 & 1,33 \\
\hline Schulter, schier, kg & 7,5 & 0,43 & 7,6 & 0,41 & 7,7 & 0,36 & 7,5 & 0,34 & 7,5 & 0,38 \\
\hline Lachs, kg & 6,5 & 0,50 & 6,7 & 0,46 & 6,8 & 0,50 & 6,4 & 0,45 & 6,4 & 0,48 \\
\hline Bauch, kg & 12,8 & 0,81 & 13,0 & 0,71 & 13,1 & 0,60 & 13,2 & 0,58 & 13,2 & 0,62 \\
\hline Bauchfleischanteil,\% & 57,0 & 3,24 & 57,7 & 3,21 & 59,1 & 3,39 & 54,1 & 3,43 & 53,7 & 3,80 \\
\hline L.PA & & & & & & & & & & \\
\hline Fleischanteil, BF, \% & 62,5 & 1,50 & 63,2 & 1,84 & 64,4 & 1,87 & 60,9 & 1,90 & 60,8 & 1,94 \\
\hline $\begin{array}{l}\text { Leitfahigkeit, Kotelett, } \\
24 \text { h p.m., mS/ }\end{array}$ & 2,93 & 0,97 & 3,78 & 1,80 & 7,64 & 2,42 & 3,01 & 0,97 & 3,59 & 1,41 \\
\hline $\begin{array}{l}\text { Tagl. Zunahme im } \\
\text { Prufabschnitt, g/Tag }\end{array}$ & 773,2 & 79,5 & 795,0 & 73,8 & 792,3 & 79,3 & 838,3 & 78,5 & 845,3 & 82,4 \\
\hline
\end{tabular}




\section{Auswertungsmethodik}

Die varianzanalytische Auswertung des Datensatzes erfolgte mit Hilfe der in Tabelle 5 beschriebenen multivariaten Mehrmerkmalsmodelle nach dem Prinzip der REMLMethode, implementiert im Programmpaket VCE 4.0 (GROENEVELD, 1996). Die Auswahl der fixen Effekte basierte auf Signifikanzergebnissen univariater Einmerkmalsanalysen mit relevanten fixen Modellfaktoren und dem zufälligen Effekt Vater. Diese Analysen wurden mit dem Programmpaket SAS (1996) und der Prozedur „Proc Mixed" durchgefuhrt.

Tabelle 5

Auswertungsmodelle der Schlachtkörpermerkmale (Anzahl Faktorstufen) (Statistical models of carcass traits (Number of effect levels))

\begin{tabular}{|c|c|c|c|c|c|c|c|c|c|c|}
\hline \multirow{2}{*}{$\begin{array}{l}\text { Datensatz } \\
\text { Faktor }\end{array}$} & \multicolumn{2}{|r|}{ BHZP } & \multicolumn{2}{|r|}{ SNW } & \multirow{2}{*}{\multicolumn{2}{|c|}{ Station Pi }} & \multicolumn{2}{|c|}{ Station Mu } & \multicolumn{2}{|c|}{ Station $\mathrm{Pi}^{*} \mathrm{Fl}$} \\
\hline & & & & & & & & & & \\
\hline $\begin{array}{l}\text { Schlachtkorper- } \\
\text { gewicht, Kov. }\end{array}$ & $\sqrt{ }$ & & $\sqrt{ }$ & & $\sqrt{ }$ & & $\sqrt{ }$ & & $\sqrt{ }$ & \\
\hline $\begin{array}{l}\text { Betrieb*Schlacht- } \\
\text { monat, fix }\end{array}$ & $\sqrt{ }$ & (24) & $\checkmark$ & (37) & & & & & & \\
\hline Geschlecht, fix & $\checkmark$ & (2) & $\sqrt{ }$ & (2) & & & & & & \\
\hline Schlachtmonat & & & & & $\checkmark$ & (21) & $\sqrt{ }$ & (21) & $\sqrt{ }$ & (21) \\
\hline LPA, fix & & & & & $\checkmark$ & (2) & $\sqrt{ }$ & (2) & $\sqrt{ }$ & (2) \\
\hline $\begin{array}{l}\text { Linie, fix } \\
\text { MHS Status, fix }\end{array}$ & $\sqrt{ }$ & $(2)^{\prime \prime}$ & & & $\sqrt{ }$ & & $\sqrt{ }$ & $(4)^{2}$ & & \\
\hline $\begin{array}{l}\text { MHS Status, fix } \\
\text { Wurf }\end{array}$ & $\sqrt{ }$ & (399) & $\sqrt{ }$ & $(563)$ & $\begin{array}{l}\sqrt{1} \\
\sqrt{1}\end{array}$ & $(4)^{63}$ & & & $\begin{array}{l}\sqrt{ } \\
\text { j }\end{array}$ & $\begin{array}{l}(2)^{4} \\
(568)\end{array}$ \\
\hline $\begin{array}{l}\text { Wurf } \\
\text { Tier }\end{array}$ & $\sqrt{ }$ & (2829) & $\sqrt{ }$ & (5006) & $\sqrt{ }$ & $\begin{array}{l}(930) \\
(6300)\end{array}$ & $\begin{array}{l}v \\
\sqrt{ }\end{array}$ & $\begin{array}{l}(669) \\
(5982)\end{array}$ & $\begin{array}{l}\sqrt{2} \\
\sqrt{ }\end{array}$ & $\begin{array}{l}(568) \\
(4056)\end{array}$ \\
\hline
\end{tabular}

Die Analysemodelle des Merkmals Alter bei Mastende der beiden Produktionsdatensätze entsprechen - mit Ausnahme des Saisoneffektes, der auf der Basis des Geburtsmonats der Schlachtschweine definiert wurde - den beschriebenen Modellen der Schlachtkörpermerkmale. Die Modelle des Merkmals Leitfähigkeit beinhalten neben den zufälligen Effekten Wurf, Tier und Fehler den Einflussfaktor Schlachttag * LPA als fixen Effekt. Insgesamt wurden bei den Datensätzen $\mathrm{Pi}, \mathrm{Mu}$ und $\mathrm{Pi}^{*} \mathrm{~F} 1$ 148, 146 bzw. 128 Schlachttage berücksichtigt. Der Faktor Schlachtkörpergewicht blieb bei der Auswertung des stationär erfassten Merkmals Tägliche Zunahme im Mastabschnitt unberiicksichtigt.

Um den Einfluss des MHS-Gens auf die geschätzten Parameter zu quantifizieren, blieb in einer zusătzlich durchgeführten Analyse der $\mathrm{Pi}$ - und $\mathrm{Pi}^{*} \mathrm{Fl}$-Stationsdaten der Modellfaktor MHS unberücksichtigt.

\section{Ergebnisse}

\section{Produktionsdaten}

Tabelle 6 beinhaltet die geschätzten Varianzverhältnisse der Merkmale der Produktjonsdatensătze. Die größte Heritabilităt besitzt in beiden Datensătzen der mít Hilfe des FOM-Sondengerätes geschätzte Fleischanteil. Mit Ausnahme des Merkmals Bauchfleischanteil befinden sich die Heritabilitäten der Auto-FOM-Schlachtkörpermerkmale auf einem niedrigeren Niveau. Unterschiede zwischen den beiden Datensätzen sind nur im Merkmal Lachs - mit erkennbar höheren Wert im Datensatz SNW - zu finden. 
Im Merkmal Alter bei Mastende ist im Datensatz BHZP eine höhere Heritabilität verbunden mit niedrigeren Wurfumwelteffekten festzustellen. Möglicherweise ist diese Beobachtung durch unterschiedliche Produktionsbedingungen in den Testbetrieben der Zuchtorganisationen oder in varianzanalytischen Problemen in der Trennung von genetischen und wurfumweltbedingten Effekten zu suchen.

Tabelle 6

Genetische Parameter für unter Produktionsbedingungen erfasste Merkmale (Genetic parameters of traits from fatting herds)

\begin{tabular}{lcccccc}
\hline Datensatz & \multicolumn{3}{c}{ BHZP } & \multicolumn{3}{c}{ SNW } \\
& $\mathrm{h}^{2}$ & $\mathrm{c}^{2}$ & $\sigma^{2}$ & $\mathrm{~h}^{2}$ & $\mathrm{c}^{2}$ & $\sigma^{2}$ \\
\hline $\begin{array}{l}\text { Merkmal } \\
\text { Fleischanteil FOM-Sonde, \% }\end{array}$ &, $29 \pm, 03$ &, $05 \pm, 02$ & 2,101 &, $43 \pm, 03$ &, $04 \pm, 01$ & 3,283 \\
$\begin{array}{l}\text { AutoFOM } \\
\quad \text { Schinken, schier, kg }\end{array}$ &, $15 \pm, 03$ &, $04 \pm, 01$ & 0,186 &, $23 \pm, 03$ &, $01 \pm, 01$ & 0,316 \\
$\quad$ Schulter, schier, kg &, $13 \pm, 03$ &, $02 \pm, 01$ & 0,015 &, $13 \pm, 02$ &, $03 \pm, 01$ & 0,017 \\
$\quad$ Lachs, kg &, $13 \pm, 03$ &, $04 \pm, 01$ & 0,025 &, $23 \pm, 02$ &, $02 \pm, 01$ & 0,044 \\
$\quad$ Bauchfleischanteil, \% &, $27 \pm, 03$ &, $03 \pm, 01$ & 4,891 &, $31 \pm, 03$ &, $03 \pm, 01$ & 6,402 \\
$\quad$ Bauch, kg &, $15 \pm, 03$ &, $01 \pm, 00$ & 0,039 &, $09 \pm, 03$ &, $05 \pm, 01$ & 0,024 \\
Alter, Tage &, $24 \pm, 03$ &, $08 \pm, 02$ & 45,163 &, $13 \pm, 01$ &, $23 \pm, 03$ & 30,571 \\
\hline
\end{tabular}

Die absoluten phänotypischen Korrelationen $\left(\mathrm{r}_{\mathrm{p}}\right)$ zwischen dem FOM-Sonden-Fleischanteil und den AutoFOM-Schlachtkörpermerkmalen liegen in beiden Datensätzen zwischen 0,44 und 0,71 (Tab. 7). Geringfügig höher liegen - mit Ausnahme der Korrelation Schulter und Lachs $\left(r_{p}=0,33\right)$ - die phänotypischen Korrelationen zwischen den AutoFOM-Merkmalen. Nur sehr geringe phänotypische Beziehungen wurden zwischen den Schlachtkörpermerkmalen und dem Alter bei Mastende sowohl im BHZPals auch SNW-Datensatz geschätzt.

Tabelle 7

Phảnotypische Korrelationen zwischen Merkmalen, die unter Produktionsbedingungen erfasst wurden. I. Zeile Datensatz SNW, 2. Zeile Datensatz BHZP (Phenotypic correlations between traits from fatting herds. $1^{\text {st }}$ row dataset SNW, $2^{\text {nd }}$ row dataset $B H Z P$ )

\begin{tabular}{|c|c|c|c|c|c|c|c|}
\hline Merkmal & & 2 & 3 & 4 & 5 & 6 & 7 \\
\hline Fleischanteil FOM-Sonde, $\%$ & 1 & $\begin{array}{l}62 \\
, 59\end{array}$ & $\begin{array}{r}49 \\
, 49\end{array}$ & $\begin{array}{l}, 47 \\
, 44\end{array}$ & $\begin{array}{l}71 \\
, 69\end{array}$ & $\begin{array}{l}-, 47 \\
-, 47\end{array}$ & $\begin{array}{l}, 10 \\
, 08\end{array}$ \\
\hline Schinken, schier, kg & 2 & & $\begin{array}{l}.63 \\
.63\end{array}$ & $\begin{array}{l}, 68 \\
, 65\end{array}$ & $\begin{array}{l}, 84 \\
, 84\end{array}$ & $\begin{array}{l}-, 56 \\
-, 55\end{array}$ & $\begin{array}{l}, 04 \\
, 03\end{array}$ \\
\hline Schulter, schier, kg & 3 & & & $\begin{array}{l}, 33 \\
, 33\end{array}$ & $\begin{array}{l}, 65 \\
, 67\end{array}$ & $\begin{array}{l}-, 57 \\
-, 57\end{array}$ & $\begin{array}{l}, 09 \\
, 07\end{array}$ \\
\hline Lachs, kg & 4 & & & & , 72 & $\begin{array}{l}-, 42 \\
-, 41\end{array}$ & $\begin{array}{l}-, 01 \\
-, 04\end{array}$ \\
\hline Bauchfleischanteil, \% & 5 & & & & & $\begin{array}{l}-, 65 \\
-, 66\end{array}$ & $\begin{array}{l}, 05 \\
, 06\end{array}$ \\
\hline Bauch, kg & 6 & & & & & & $\begin{array}{r}-, 05 \\
-, 06\end{array}$ \\
\hline Alter, Tage & 7 & & & & & & \\
\hline
\end{tabular}

Die geschätzten genetischen Beziehungen zwischen den AutoFOM-Merkmalen liegen 
in beiden Datensätzen in der Regel deutlich über 0,9 (Tab. 8). Auf einem ähnlich hohen Niveau liegen die genetischen Korrelationen zwischen den AutoFOM- und FOMSondenmerkmalen im SNW-Datensatz, während im BHZP-Datensatz insbesondere zwischen dem Merkmal Lachs und FOM-Sonde vergleichsweise niedrigere Beziehungen geschätzt wurden.

Auf deutlich niedrigerem Niveau wurden die Beziehungen zwischen den Schlachtkörpermerkmalen und dem Alter bei Mastende geschätzt. Vergleicht man die beiden Datensätze, so fallen die unterschiedlichen Vorzeichen dieser Merkmalsbeziehungen auf. Ein frühes Mastende bei gegebenem Mastendgewicht lässt sich durch eine hohe Futteraufnahme und/oder effiziente Futterverwertung erreichen. Eine effiziente Futterverwertung ist bei einem hohen Proteinansatzvermögen und damit verbunden verbesserter Schlachtkörperqualität zu erwarten. Zwischen Futteraufnahme einerseits und Futterverwertung bzw. Schlachtkörperqualität andererseits wurden in der Literatur züchterisch unerwünschte Beziehungen erwähnt, die sich u. a. durch hormonelle Regelmechanismen erklären lassen. Die Frage, ob rnit verbesserter Schlachtkörperqualităt sich mehr die Futteraufnahme reduziert oder sich die Futterverwertung stärker verbessert, ist vermutlich bei den beiden Produktionsdatensätzen unterschiedlich zu beantworten.

Tabelle 8

Genetische Korrelationen zwischen Merkmalen, die unter Produktionsbedingungen erfasst wurden. 1. Zeile Datensatz SNW, 2. Zeile Datensatz BHZP (Genetic correlations between traits from fatting herds. $1^{\text {st }}$ row dataset SNW, $2^{\text {nd }}$ row dataset $\mathrm{BHZP}$ )

\begin{tabular}{|c|c|c|c|c|c|c|c|}
\hline Merkmal & & 2 & 3 & 4 & $s$ & 6 & 7 \\
\hline Fleischanteil FOM-Sonde, $\%$ & 1 & $\begin{array}{l}96 \\
, 82\end{array}$ & $\begin{array}{l}, 99 \\
, 87\end{array}$ & $\begin{array}{l}, 88 \\
, 67\end{array}$ & $\begin{array}{l}98 \\
, 83\end{array}$ & $\begin{array}{r}-, 98 \\
-, 75\end{array}$ & $\begin{array}{r}-, 20 \\
, 13\end{array}$ \\
\hline Schinken, schier, kg & 2 & & $\begin{array}{l}96 \\
, 97\end{array}$ & $\begin{array}{l}, 97 \\
, 89\end{array}$ & $\begin{array}{l}99 \\
, 99\end{array}$ & $\begin{array}{r}-, 91 \\
-, 94\end{array}$ & $\begin{array}{r}-, 13 \\
.28\end{array}$ \\
\hline Schulter, schier, kg & 3 & & & $\begin{array}{l}.87 \\
.72\end{array}$ & $\begin{array}{l}98 \\
, 99\end{array}$ & $\begin{array}{r}-, 95 \\
-, 99\end{array}$ & $\begin{array}{r}-19 \\
, 21\end{array}$ \\
\hline Lachs, kg & 4 & & & & $\begin{array}{l}95 \\
, 84\end{array}$ & $\begin{array}{l}-, 80 \\
-77\end{array}$ & $\begin{array}{l}, 00 \\
.04\end{array}$ \\
\hline Bauchfleischanteil, \% & $s$ & & & & & $\begin{array}{l}95 \\
-97\end{array}$ & $\begin{array}{r}-, 14 \\
, 22\end{array}$ \\
\hline Bauch, $\mathrm{kg}$ & 6 & & & & & & $\begin{array}{r}.15 \\
-, 07\end{array}$ \\
\hline Alter, Tage & 7 & & & & & & \\
\hline Standardfehler & &, 02 & $\begin{array}{l}, 02 \\
, 06\end{array}$ & $\begin{array}{l}, 05 \\
10\end{array}$ & $\begin{array}{l}, 00 \\
, 04\end{array}$ & $\begin{array}{l}, 00 \\
, 09\end{array}$ & $\begin{array}{l}, 14 \\
23\end{array}$ \\
\hline
\end{tabular}

\section{$\underline{\text { Stationsdaten }}$}

Bei allen analysierten Herkünften wurden hohe Heritabilitäten für die Merkmale Fleischanteil nach Bonner Formel und FOM-Sonde geschätzt (Tab. 9). Die geschätzten Erblichkeitsgrade der AutoFOM-Merkmale liegen bei den Mutterassen und Pi*FlSchweinen auf einem geringfügig niedrigerem Niveau. Deutlich reduziert hingegen sind die geschätzten Heritabilitäten der AutoFOM-Merkmale bei der Rasse Pi. Sie erreichen bei keinem dieser Merkmale $20 \%$. Darüber hinaus führt bei Rasse Pi die 
Berücksichtung des Faktors MHS erwartungsgemäß zu einer deutlichen Verringerung der geschätzten genetischen Varianzen, mit Heritabilitäten von weniger als $10 \%$. Es ist demzufolge zu vermuten, dass nach der angestrebten Elimination des MHS-Stressgens $\mathrm{P}$ eine zufriedenstellende genetische Fundierung von AutoFOM-Merkmalen bei der Rasse Pi nicht gegeben ist. Verantwortlich hierfür könnte eine Einschränkung der genetischen Varianz von Schlachtkörpermerkmalen - verursacht durch die langandauernde intensive Selektion auf diesen Merkmalskomplex - sein. Die hohen Heritabilitäten des geschätzten Fleischanteils nach Bonner Formel und FOM-Sonde widersprechen allerdings dieser Hypothese. Die Entwicklung des AutoFOM-Gerätes orientiert sich hinsichtlich der Schlachtkörperqualität an dem Niveau von Produktionstieren. Es ist somit zu bezweifeln, ob die Messgenauigkeit des AutoFOM-Gerätes bei einem Fleischanteil des Schlachtkörpers von deutlich mehr als $65 \%$ ausreicht, um sinnvolle Selektionsentscheidungen treffen zu können. Bei den Mutterlinien und Pi*F1-Schweinen hingegen stellen die AutoFOM-Meßergebnisse möglicherweise eine sinnvolle Alternative zur aufwendigen LPA-Schlachtkörperbeurteilung dar.

Tabelle 9

Genetische Parameter für stationăr erfasste Merkmale. 1. Zeile Modell ohne fixen Modelifaktor MHS, 2. Zeile Modell mit fixem Modellfaktor MHS. (Genetic parameters for traits from station test, $1^{\text {st }}$ row model without the fixed effect MHS, $2^{\text {nd }}$ row model with fixed effect $M H S$ )

\begin{tabular}{|c|c|c|c|c|c|c|c|c|c|}
\hline \multirow{2}{*}{$\begin{array}{l}\text { Herkunft } \\
\text { Merkmal }\end{array}$} & \multicolumn{3}{|c|}{ Pi } & \multicolumn{3}{|c|}{$\mathrm{Mu}$} & \multicolumn{3}{|c|}{$\mathrm{Pi}^{*} \mathrm{~F} 1$} \\
\hline & $\mathrm{h}^{2}$ & $c^{2}$ & $\sigma^{2}$, & $\mathrm{h}^{2}$ & $c^{2}$ & $\sigma^{2}$ & $h^{2}$ & $\mathrm{c}^{2}$ & $\sigma^{2}$ \\
\hline \multirow{2}{*}{$\begin{array}{l}\text { Fleischanteil, } \\
\text { Bonner Formel, \% }\end{array}$} & .70 &, 09 & 2,583 & ,64 &, 09 & 2,674 &, $6 \mathrm{I}$ & ,0I & 2,144 \\
\hline &, 41 &, 17 & 1,289 & & & &, 62 &, 01 & 2,154 \\
\hline \multirow{2}{*}{$\begin{array}{l}\text { Fleischanteil, } \\
\text { FOM-Sonde, \% }\end{array}$} &, 65 &, 03 & 2,879 &, 58 &, 12 & 6,434 & .47 &, 03 & 2,521 \\
\hline & 37 &, 09 & 1,360 & & & & $A 8$ &, 04 & 2,548 \\
\hline \multirow[t]{2}{*}{ Schinken, schier, kg } &, 15 & .04 & 0,034 &, 40 &, 07 & 0,181 &, 19 & .27 & 0,230 \\
\hline &, 07 &, 05 & 0,016 & & & &, 19 &, 27 & 0,234 \\
\hline \multirow[t]{2}{*}{ Lachs, kg } &, 14 & ,03 & 0,007 &, 42 &, 01 & 0,022 & 20 &, 04 & 0,032 \\
\hline & .09 &, 05 & 0,004 & & & & 21 &, 04 & 0,033 \\
\hline \multirow[t]{2}{*}{ Schulter, schier, kg } & .04 &, 07 & 0,001 & 27 & $\sqrt{10}$ & 0,013 &, 23 & ,01 & 0,018 \\
\hline &, 02 &, 07 & 0,001 & & & &, 23 &, 01 & 0,018 \\
\hline \multirow[t]{2}{*}{ Bauchfleischanteil, \% } & .19 &, 08 & 2,107 &, 59 &, 03 & 15,51 &, 40 &, 03 & 5,217 \\
\hline &, 10 & 10 & 1,029 & & & &, 40 &, 13 & 5,248 \\
\hline \multirow[t]{2}{*}{ Bauch, kg } &, 06 &, 05 & 0,004 &, 30 &, 04 & 0,032 &, 05 &, 00 & 0,009 \\
\hline &, 06 &, 05 & 0,004 & & & &, 06 &, 00 & 0,010 \\
\hline \multirow{2}{*}{$\begin{array}{l}\text { Taggl. Zunahme im } \\
\text { Prllfabschnitt }\end{array}$} &, 48 & .12 & 2587 &, 46 &, 11 & 3473 & \$5 &, 05 & 3198 \\
\hline &, 28 &, 12 & 2630 & & & & .55 &, 05 & 3200 \\
\hline \multirow{2}{*}{$\begin{array}{l}\text { Leitfahigkeit, Kotelett, } \\
24 \text { h p.m. }\end{array}$} & 92 &, 00 & 7,026 &, 21 &, 01 & 0,120 & 64 &, 01 & 1,003 \\
\hline &, 12 & ,16 & 0,486 & & & & .47 & .04 & 0,690 \\
\hline \multirow[t]{2}{*}{ Standardfehler } & 01 &, 00 & &, 06 &, 02 & &, 02 & .02 & \\
\hline &, 08 &, 03 & &, 08 &, 04 & & 08 &, 04 & \\
\hline
\end{tabular}

Die absoluten phänotypischen (Tab. 10) und genetischen Beziehungen (Tab. 11) sowohl zwischen den AutoFOM-Schlachtkörpermerkmalen als auch zwischen den Au- 
toFOM-Merkmalen und dem Fleischanteil nach Bonner Formel liegen bei allen Herkünften überwiegend auf einem sehr hohen Niveau. Hiervon ausgenommen sind die vergleichsweise losen Beziehungen zwischen dem Merkmal Bauchgewicht und den übrigen Merkmalen bei der Rasse Pi. Die niedrige Heritabilităt des Bauchgewichts bei der Rasse Pi ist bei der Interpretation dieses Ergebnisses zu berlcksichtigen.

Tabelle 10

Phänotypische Korrelationen zwischen stationär erfassten Merkmalen. I. Zeile Datensatz Pi, 2. Zeile Datensatz $\mathrm{Mu}$, 3. Zeile Datensatz $\mathrm{Pi}{ }^{中} \mathrm{~F} 1$ (Phenotypic correlations between traits from station test. $1^{\text {"t }}$ row dataset $\mathrm{Pi}, 2^{\text {nd }}$ row dataset $\mathrm{Mu}, 3^{\text {rd }}$ row dataset $\mathrm{Pi}{ }^{*} \mathrm{~F} 1$ )

\begin{tabular}{|c|c|c|c|c|c|c|c|c|}
\hline Merkmal & & 2 & 3 & 4 & 5 & 6 & 7 & 8 \\
\hline $\begin{array}{l}\text { Fleischanteil, } \\
\text { Bonner Formel }\end{array}$ & (1) & $\begin{array}{l}, 72 \\
, 83 \\
, 77\end{array}$ & $\begin{array}{l}.40 \\
.72 \\
.50\end{array}$ & $\begin{array}{l}, 34 \\
, 62 \\
, 43\end{array}$ & $\begin{array}{l}.23 \\
.55 \\
, 36\end{array}$ & $\begin{array}{l}.51 \\
.78 \\
.63\end{array}$ & $\begin{array}{l}-, 11 \\
-, 58 \\
-, 25\end{array}$ & $\begin{array}{l}-, 21 \\
, 01 \\
-, 19\end{array}$ \\
\hline $\begin{array}{l}\text { Fleischanteil, } \\
\text { FOM-Sonde }\end{array}$ & (2) & & $\begin{array}{l}.40 \\
, 66 \\
, 48\end{array}$ & $\begin{array}{l}.33 \\
.59 \\
.44\end{array}$ & $\begin{array}{l}.28 \\
.51 \\
.46\end{array}$ & $\begin{array}{l}.53 \\
.73 \\
.65\end{array}$ & $\begin{array}{l}-.11 \\
-.55 \\
-.31\end{array}$ & $\begin{array}{l}-, 12 \\
-, 00 \\
-, 12\end{array}$ \\
\hline Schinken, schier & (3) & & & $\begin{array}{l}, 68 \\
, 67 \\
, 54\end{array}$ & $\begin{array}{l}, 54 \\
.64 \\
.54\end{array}$ & $\begin{array}{l}, 74 \\
, 85 \\
.71\end{array}$ & $\begin{array}{l}-, 06 \\
-, 61 \\
-, 32\end{array}$ & $\begin{array}{l}-, 07 \\
, 06 \\
, 03\end{array}$ \\
\hline Lachs & (4) & & & & $\begin{array}{l}, 27 \\
, 42 \\
, 29\end{array}$ & $\begin{array}{l}, 72 \\
, 76 \\
71\end{array}$ & $\begin{array}{l}-, 06 \\
-, 50 \\
-, 34\end{array}$ & $\begin{array}{r}-, 05 \\
, 08 \\
-, 01\end{array}$ \\
\hline Schulter, schier & (5) & & & & & $\begin{array}{l}, 39 \\
, 70 \\
, 58\end{array}$ & $\begin{array}{l}, 03 \\
-, 62 \\
-, 33\end{array}$ & $\begin{array}{l}-, 10 \\
, 02 \\
-, 03\end{array}$ \\
\hline Bauchfleischanteil & (6) & & & & & & $\begin{array}{l}-, 39 \\
-, 69 \\
-, 50\end{array}$ & $\begin{array}{r}-, 10 \\
, 05 \\
-, 08\end{array}$ \\
\hline Bauch & (7) & & & & & & & $\begin{array}{l}, 05 \\
, 01 \\
.10\end{array}$ \\
\hline
\end{tabular}

Tagl. Zunahme im

Die herkunftsspezifischen Beziehungen zwischen dem Merkmal tägliche Zunahme im Prüfungsabschnitt und den Schlachtkörpermerkmalen sind zum Teil widersprïchlich. Diese Beziehungen sind bei den Herkünften $\mathrm{Pi}$ und $\mathrm{Pi}^{*} \mathrm{~F} 1$ antagonistisch ausgeprägt, während züchterisch erwünschte Beziehungen bei den Mutterlinien festzustellen sind. Wie bereits bei der Interpretation der Feldprüfergebnisse erwähnt, sind die wechselseitigen Beziehungen zwischen Futterverwertung, Futteraufnahme und Schlachtkörperqualität zur Erklärung der unterschiedlichen genetischen Korrelationsausprägung heranzuziehen.

Die genetische Beziehung zwischen den Fleischbeschaffenheits- und Schlachtkörpermerkmalen ist bei allen Herkünften züchterisch negativ ausgeprägt. Eine Berücksichtigung des Modellfaktors MHS-Status führt zwar bei der Rasse Pi zu einer Lockerung dieser unerwünschten Beziehung (Tab. 12); die geschätzten Korrelationen bei den Mutterlinien zeigen jedoch, dass trotz erfolgreicher MHS-Sanierung, die züchterische 
Verbesserung der Schlachtkörperqualität nach wie vor eine Verschlechterung der Fleischbeschaffenheit zur Folge hat.

Tabelle 11

Genetische Korrelationen zwischen stationär erfassten Merkmalen. 1. Zeile Datensatz Pi, 2. Zeile Datensatz Mu, 3. Zeile Datensatz $\mathrm{Pi}^{*} \mathrm{~F} 1$ (Genetic correlations between traits from station test. $\mathrm{I}^{\text {st }}$ row dataset $\mathrm{Pi}, 2^{\text {nd }}$ row datasel $\mathrm{Mu}, 3^{\text {rd }}$ row dataset $\mathrm{Pi}^{*} \mathrm{~F} 1$ )

\begin{tabular}{|c|c|c|c|c|c|c|c|c|}
\hline Merkmal & & 2 & 3 & 4 & 5 & 6 & 7 & 8 \\
\hline $\begin{array}{l}\text { Fleischanteil, } \\
\text { Bonner Formel, \% }\end{array}$ & (1) & $\begin{array}{l}98 \\
96 \\
, 86\end{array}$ & $\begin{array}{l}99 \\
96 \\
97\end{array}$ & $\begin{array}{l}75 \\
.97 \\
.81\end{array}$ & $\begin{array}{l}, 99 \\
, 92 \\
, 77\end{array}$ & $\begin{array}{l}97 \\
, 93 \\
.86\end{array}$ & $\begin{array}{l}-, 35 \\
-, 88 \\
-, 99\end{array}$ & $\begin{array}{r}-, 10 \\
, 24 \\
-, 51\end{array}$ \\
\hline $\begin{array}{l}\text { Fleischanteil, } \\
\text { FOM-Sonde, \% }\end{array}$ & (2) & & $\begin{array}{l}99 \\
.88 \\
, 99\end{array}$ & $\begin{array}{l}, 82 \\
, 99 \\
, 90\end{array}$ & $\begin{array}{l}99 \\
, 80 \\
.95\end{array}$ & $\begin{array}{l}99 \\
90 \\
, 99\end{array}$ & $\begin{array}{r}-, 12 \\
-, 90 \\
-, 99\end{array}$ & $\begin{array}{r}, 01 \\
, 02 \\
-, 53\end{array}$ \\
\hline Schinken, schier, kg & (3) & & & $\begin{array}{l}95 \\
99 \\
99\end{array}$ & $\begin{array}{l}99 \\
98 \\
99\end{array}$ & $\begin{array}{l}92 \\
, 99 \\
99\end{array}$ & $\begin{array}{r}-, 19 \\
-, 99 \\
-, 99\end{array}$ & $\begin{array}{r}-, 01 \\
, 35 \\
-, 78\end{array}$ \\
\hline Lachs, kg & (4) & & & & $\begin{array}{l}, 99 \\
.99 \\
.76\end{array}$ & $\begin{array}{l}, 86 \\
, 96 \\
, 95\end{array}$ & $\begin{array}{l}-, 29 \\
-, 94 \\
-, 99\end{array}$ & $\begin{array}{r}, 03 \\
, 33 \\
-, 45\end{array}$ \\
\hline Schulter, schier, kg & $(5)$ & & & & & $\begin{array}{l}, 99 \\
, 99 \\
, 93\end{array}$ & $\begin{array}{l}-, 89 \\
-, 97 \\
-, 99\end{array}$ & $\begin{array}{r}-, 62 \\
, 36 \\
-, 68\end{array}$ \\
\hline Bauchfleischanteil, \% & $(6)$ & & & & & & $\begin{array}{r}-, 28 \\
-, 97 \\
-, 99\end{array}$ & $\begin{array}{r}, 09 \\
, 30 \\
-, 62\end{array}$ \\
\hline Bauch, kg & (7) & & & & & & & $\begin{array}{r}.27 \\
-47 \\
.86\end{array}$ \\
\hline
\end{tabular}

Tagl. Zunahme im

(8)

\begin{tabular}{|c|c|c|c|c|c|c|c|c|}
\hline Standardfehler & $\begin{array}{l}\text { von } \\
\text { bis }\end{array}$ & $\begin{array}{l}, 02 \\
, 03\end{array}$ & $\begin{array}{l}, 00 \\
, 07\end{array}$ & $\begin{array}{l}00 \\
11 \\
\end{array}$ & $\begin{array}{l}00 \\
15\end{array}$ & $\begin{array}{l}, 00 \\
.07\end{array}$ & $\begin{array}{r}.00 \\
.56 \\
\end{array}$ & $\begin{array}{r}, 02 \\
, 90 \\
\end{array}$ \\
\hline
\end{tabular}

\section{Schlussfolgerungen}

\section{Zuchtwertschätzung (Top-Genetik) fulr Besamungseber}

Die AutoFOM-Merkmale sind seit Beginn des Jahres 2000 im nordwestdeutschen Raum Grundlage des Bezahlungssystems (BEUCK, 1999). BRANSCHEIDT et al. (1997) zeigten in ihren Untersuchungen, dass der wahre Fleischanteil der Schlachtkörper mit dem AutoFOM-Klassifizierungssystem in zufriedenstellender Weise erfasst werden kann. Die ökonomische Relevanz der AutoFOM-Merkmale ist demnach unbestritten. Daruber hinaus ist - wie die oben genannten Untersuchungsergebnisse verdeutlichen - eine ausreichende genetische Fundierung der AutoFOM Prüfergebnisse von Produktionsschweinen gegeben.

Unter diesen Rahmenbedingungen hat sich die Besamungsstation GFS Ascheberg entschlossen, die im Rahmen der Feldprifung erhobenen AutoFOM Merkmale zur genetischen Rangierung der Besamungseber zu nutzen. Auf der Basis der geschătzten ge- 
Tabelle 12

Genetische (rg) und phänotypische (rp) Korrelationen zwischen stationăr erfassten Schlachtkorpermerkmalen und der Leitfahigkeit, m. longisimus dorsi, 24 h p.m, mit $(+)$ und ohne $(-)$ Berllcksichtigung des Modellfaktors MHS (Genetic $(\mathrm{rg})$ and phenotypic $(\mathrm{rp})$ correlations between carcass traits from station test and conductivity measured in the $\mathrm{m}$. longisimus dorsi, $24 \mathrm{~h}$ p.m. with $(+)$ and without $(-)$ consideration of the model factor MHS)

\begin{tabular}{|c|c|c|c|c|c|c|c|c|}
\hline \multirow{3}{*}{$\begin{array}{l}\text { Herkunft } \\
\text { Merkmal } \\
\text { Modell } \\
\end{array}$} & \multicolumn{4}{|c|}{$\mathrm{Pi}$} & \multicolumn{4}{|c|}{$\mathrm{Pi}+\mathrm{Fl}$} \\
\hline & \multicolumn{2}{|c|}{ Ip } & \multicolumn{2}{|c|}{$\mathrm{rg}$} & \multicolumn{2}{|c|}{ rp } & \multicolumn{2}{|c|}{$\mathrm{rg}$} \\
\hline & -MHS & $+\mathrm{MHS}$ & -MHS & $+\mathrm{MHS}$ & -MHS & $+\mathrm{MHS}$ & -MHS & $+\mathrm{MHS}$ \\
\hline Fleischanteil, BF &, 34 &, 18 &, 53 &, 30 &, 23 &, 22 & .26 &, 38 \\
\hline Fleischanteil, FOM &, 37 &, 19 & ,55 & 02 & .17 &, 17 &, 32 &, 43 \\
\hline Schinken, schier & 20 &, 13 & 47 &,- 27 &, 14 &, 11 &, 46 &, 54 \\
\hline Lachs &, 13 & ,09 &, 27 & 21 &, 12 &, 12 &, 50 &, 67 \\
\hline Schulter, schier &, 14 &, 08 &, 87 &, 02 &, 13 &, 06 &, 13 &, 16 \\
\hline Bauchfleischanteil & 20 &, 10 &, 44 &,- 26 &, 16 & 10 & .18 &, 29 \\
\hline Bauch & 02 &,- 00 &, 27 &.,- 17 &,- 12 &,- 04 &,- 59 &,- 97 \\
\hline $\begin{array}{l}\text { Taggl. Zunahme im Prafab- } \\
\text { schnitt }\end{array}$ &,- 03 &,- 07 & ,05 & -.22 &,- 05 & -13 &, 18 &, 11 \\
\hline Standardfehler & & & $\begin{array}{l}.06 \\
.39\end{array}$ & $\begin{array}{l}, 10 \\
, 34\end{array}$ & & & $\begin{array}{l}.08 \\
, 70\end{array}$ & $\begin{array}{l}, 00 \\
, 17\end{array}$ \\
\hline
\end{tabular}

netischen Parameter wurde ein entsprechendes Zuchtwertschätzmodell für die beiden Zuchtorganisationen BHZP und SNW entwickelt (BRANDT und THOLEN, 2000). In Zukunft sollen für alle Endprodukteber die Zuchtwerte für die in Tabelle 13 aufgeführten Einzelmerkmale ausgewiesen werden. Das multivariate Auswertungsmodell beinhaltet die bei der Parameterschätzung dargestellten Einflussfaktoren (Tab, 5). Die angegebene relative Gewichtung der AutoFOM-Merkmale entspricht dem derzeitigen Handelswert der jeweiligen Teilstücke.

Tabelle 13

Merkmale und deren relative Gewichtung in der Zuchtwertschatzung von Besamungsebern (Traits and their relative weightings in the estimation of breeding value of Al boars)

\begin{tabular}{lc}
\hline Merkmale & Relative Gewichtung filr den Gesamtzuchtwert \\
\hline FOM-Muskelfleischanteil (Sonde) & 100 \\
AutoFOM, Lachs, kg & 70 \\
AutoFOM, Schinken, kg & 55 \\
AutoFOM, Schulter, kg & \\
AutoFOM Bauchfleischanteil, \% & 5 \\
Bauchgewicht, kg & $25-35$ (abhăngig vom Bauchfleischanteil) \\
Alter bei Mastendgewicht, Tag & 5 \\
\hline
\end{tabular}

In der Übergangsphase wird man im nordwestdeutschen Raum eine Mischsituation vorfinden, in der Form, dass von den Besamungsebern teilweise Nachkommen mit AutoFOM und/oder FOM-Sonde-Messungen vorliegen werden. Es wird in der Umstellungsphase auch weiterhin Produzenten geben, die entweder nach AutoFOM oder FOM vermarkten. Daher erscheint es notwendig, für alle Eber Zuchtwerte für beide Klassifizierungssysteme zu schätzen.

Das von BEUCK (1999) vorgeschlagene Abrechnungssystem besitzt als wichtige Komponenten Ober- und Untergrenzen für die Teilstücke Schinken und Lachs. Folglich ist die produktionstechnische Steuerung des Mastendgewichts von großer Bedeu- 
tung. Der Zuchtwert Alter bei durchschnittlichem Mastendgewicht zeigt züchterische Wege auf, die Mastdauer und das Mastendgewicht zu beeinflussen. Die relative Gewichtung des Alters entspricht einem Grenznutzen von ca. 0,45 DM pro Tag.

Für die Eberrangierung der TOP-Genetik-Programme wird der Gesamtzuchtwert herangezogen, der auf ein Mittel von 100 und eine Standardabweichung von 20 Punkten standardisiert wird. Neben den Naturalzuchtwerten werden auch die Anzahl an Nachkommen eines jeden Ebers angegeben, von denen der FOM-Magerfleischanteil bzw. AutoFOM-Ergebnisse vorliegen. Zusätzlich zu den tabellarisch aufgeführten Zuchtwerten (Gesamtindex, naturale Teilzuchtwerte) werden alle Einzelzuchtwerte relativ zueinander (in Einheiten der genetischen Standardabweichung) in Form eines Balkendiagramms dargestellt (Abb.). Die Darstellung des Merkmals Alter bei Mastende erfolgt im züchterischen Sinne. Mit Hilfe dieser Graphiken wird dem Produzenten die Möglichkeit gegeben, Eber auszuwählen, die besondere Überlegenheit in einzelnen Merkmalen zeigen, und damit eine eigene Gewichtung eines Gesamtzuchtwertes vornehmen zu können.
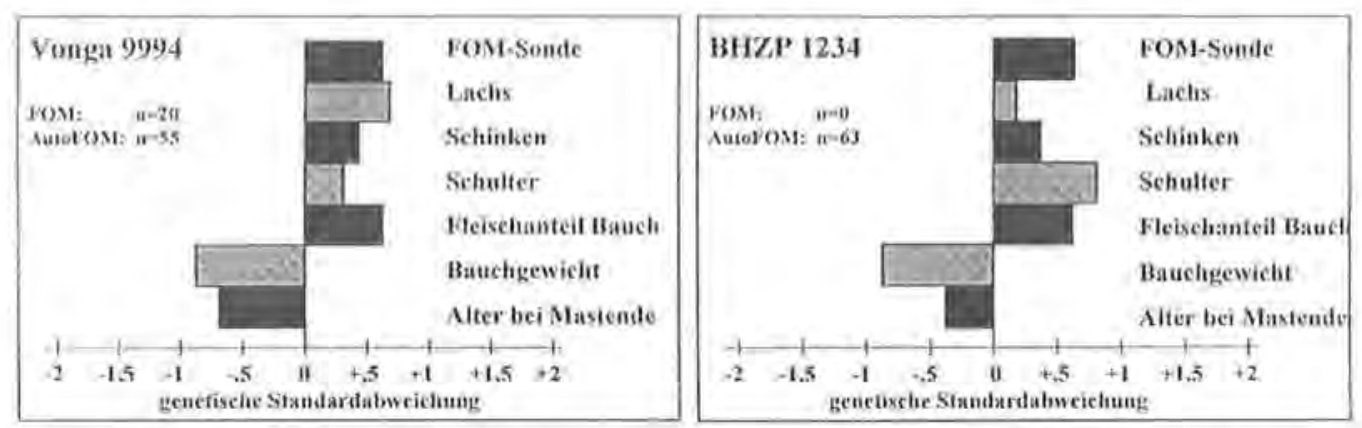

Abb.: Balkendiagramme der Zuchtwertschatzergebnisse fur Besamungseber (Diagrams of estimated breeding values of AI-Boars)

\section{Stationsprilfung}

Die züchterischen Aktivitäten in den Basiszuchtbetrieben der nordrhein-westfälischen Herdbuchbetriebe basieren auf den Ergebnissen der stationär durchgeführten Leistungsprüfung. In der vorliegenden Untersuchung wurden straffe genetische Beziehungen zwischen dem stationär erfassten Merkmal Fleischanteil Bonner Formel und den AutoFOM-Merkmalen geschätzt. Das stationär erfasste Merkmal Fleischanteil Bonner Formel ist demzufolge erkennbar geeignet, die AutoFOM-Merkmale züchterisch zu verbessern. Díe Zuverlässigkeit der AutoFOM-Merkmale zur Beurteilung des Handelswertes wurde in der Untersuchung von BRANSCHEID et al. (1997) bestätigt. Daruber hinaus ist bei den Herkunften $\mathrm{Pi}^{*} \mathrm{~F} 1$ sowie den Mutterlinien eine ausreichende genetische Fundierung gegeben, so dass aus Kostengründen das AutoFOMMeßprinzip als Alternative der aufwendigen Stationsprüfung angesehen werden kann. Nachteilig an einer solchen Vorgehensweise ist jedoch die Abhängigkeit der objektiven Leistungsprüfung von dem Anbieter automatisierter Klassifizierungsgeräte. Eine längerfristig, nach festgelegten Richtlinien kontinuierlich durchgeführte Leistungsprüfung kann demnach nicht garantiert werden. 
Die Ergebnisse unserer Untersuchungen zeigen, dass die AutoFOM-Merkmale bei den Pi-Reinzuchttieren deutlich niedrigere Erblichkeitsgrade aufweisen. Möglicherweise ist die genetische Variation aufgrund der intensiven Selektion der Pi-Eber bereits eingeschränkt. Wahrscheinlicher ist jedoch, dass die Genauigkeit des AutoFOM-Gerätes bei Magerfleischanteilen von mehr als $65 \%$ für Selektionsentscheidungen nicht mehr ausreicht. Hieraus folgt, dass eine Verbesserung der Schlachtkörperqualität bei den PiReinzuchtebern zur Zeit nur mit Hilfe von stationären Prüfergebnissen möglich erscheint.

Einschränkend sei jedoch darauf hingewiesen, dass die s.g. Bonner Formel zur Abschätzung des Fleischanteils vor ca. 15 Jahren entwickelt wurde. Inzwischen hat sich sowohl die Prüfmethodik und (Erhöhung des Schlachtendgewichts von $80 \mathrm{~kg}$ auf 85 $\mathrm{kg}$, Prüfung von Börgen bei den Mutterlinien) als auch der Genotyp der marktüblichen Herkünfte wesentlich verändert, so dass eine Überprüfung dieser Formel zur Abschätzung des wahren Fleischanteils bzw. Handelswertes notwendig erscheint.

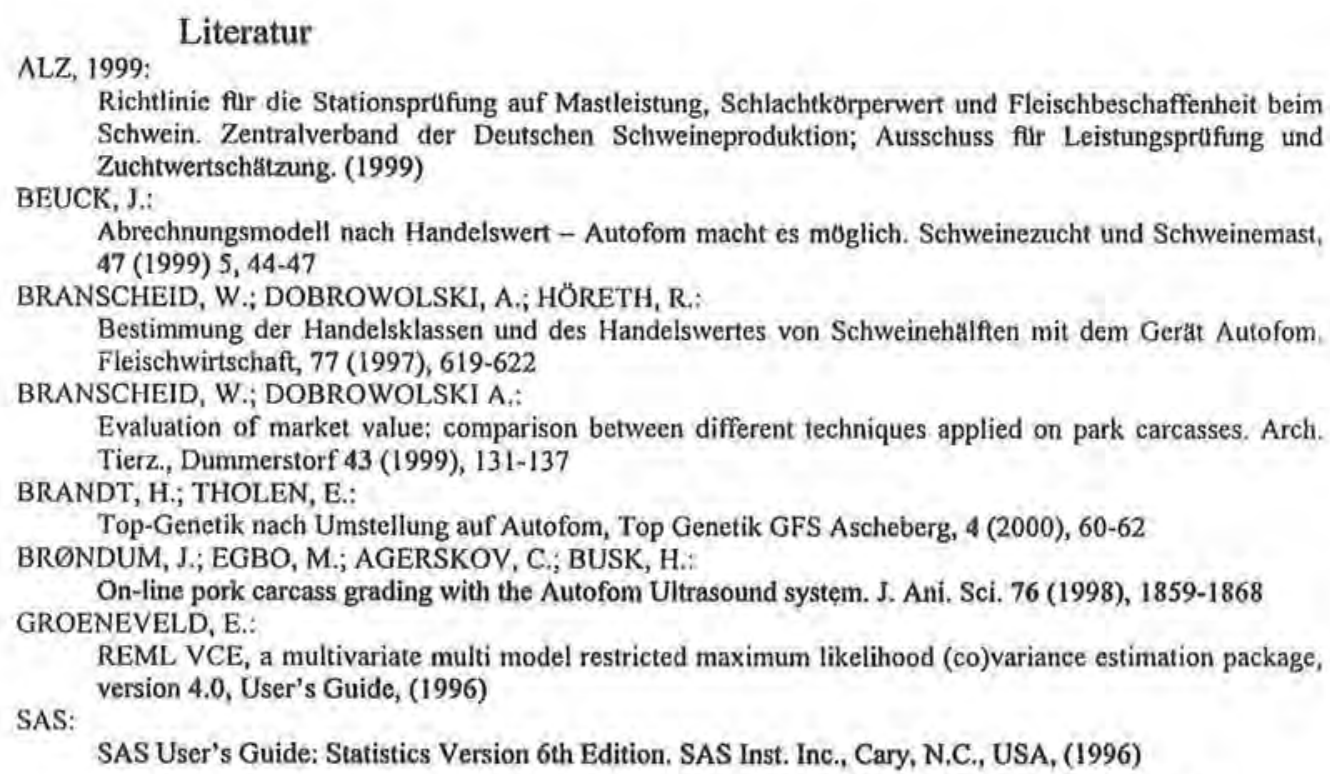

Eingegangen: 23.08 .2000

Akzeptiert: $16.11,2000$

Anschriften der Verfasser

Dr. ERNST THOLEN, Prof. Dr, KARL SCHELLANDER

Institut fur Tierzuchtwissenschaft der

Rheinischen Friedrich-Wilhelms-Universităt Bonn

Endenicher Allee is

D-53115 Bonn

Dr. HUBERT HENNE

Zuchtungszentrale

Deutsches Hybrid-

schwein

Postfach 3040

PD Dr. HORST BRANDT

Institut fur Tierzucht und Haustiergenetik

D-21320 Laneburg

Der Universitast Gottingen

Dr. FRANZ-JOSEF STORK

Albrechi-Thaer-Weg 3

Schweinezüchterverband

D-37075 Göttingen

Nord-West

Engelstraße 50

D-48143 Munster 


\section{Buchbesprechung}

\section{Schweineproduktion 1999 in Deutschland (Ausgabe 2000)}

Bearb. von: EVA MARIA GATZKA, KLEMENS SCHULZ, DR. JENS INGWERSEN

Hrsg.: Zentralverband der Deutschen Schweineproduktion e.V. (ZDS), Bonn, 2000

164 Seiten, 81 Tabellen, ISSN 0179-1001, I7,-DM

Mit dieser 47. Ausgabe der bewahrten Jahresdokumentation informiert der Zentralverband der Deutschen Schweineproduktion Uber die aktuelle Entwicklung der Schweineproduktion in Deutschland und der Europalschen Gemeinschat sowie den Leistungsstand der organisierten deutschen Schweinezucht und -produktion. Es stimmt hoffnungsvoll, dass trotz des noch nicht uberwundenen Preistiefs, was sich besonders in den FerkeJ-aber auch den Schlachttierpreisen ausdruckt, ein merkbarer Anstieg der Schweineschlachtungen aber auch des Schweinefleischverbrauches/Kopf bei gesunkenen Verbraucherpreisen abzeichnete. Fortgesetzt hat sich der Rückgang in der Anzahl der Schweine haltenden Betriebe bei gleichzeitigem Anstieg der Anzahl gehaltener Schweine bzw. Zuchtsauen je Betrieb. Als Ausdruck der Marktentwicklung ein erwarteter, zunehmender KonzentrationsprozeB, verursacht durch steigenden Kostendruck auf die Betriebe. Sowohl die Buchabschnitte zur Leistungsprifung der verschiedenen Rassen und Kreuzungen als auch der Berichterstattung lber die Ergebnisse der Arbeit der Erzeugerringe, dokumentieren den hohen Leistungsstand, welcher in der deutschen Schweinezucht und -produktion erreicht wurde. Den Realitaten Rechnung tragend wurde in diesen Bericht neu der Abschnitt Uber die Zuchtuntemehmen und sonstigen Zuchtorganisationen aufgenommen. Die im letzten Buchteil enthaltenen vergleichenden Analysen, insbesondere die Ergebnisse der Wirtschaftlichkeitskontrolle bei Ferkeln und Mastschweinen, verdeutlichen die Notwendigkeit Wege zur weiteren betrieblichen Kosteneinsparungen zu suchen und durch Nutzung inner- und uberbetrieblicher Reserven, die Wirtschaftlichkeit der Schweinehaltung zu verbessern.

In abersichtlichen Tabellen wurde ein sehr umfangreiches und informatives Datenmaterial zusammengestellt. Es vermittelt Informationen zu folgenden Komplexen:

- Markt fur Schweine und Schweinefleisch in Deutschland

- Markt fur Schweine und Schweinefleisch in der EU und Drittlandern

- Produktion von Schweinen in Deutschland

- Produktion von Schweinen und Schweinefleisch in der EU und Drittlăndern

- Schweinezucht in Deutschland

- Zuchtunternehmen und sonstige Zuchtorganisationen

- Leistungsprufungen in der Schweinezucht

- Kunstliche Besamung beim Schwein

- Schweineerzeugerringe in Deutschland

Dem Tabellenwerk ist eine in deutscher, englischer und franzosischer Sprache verfaßte tabellarische Kurzinformation der wichtigsten Buchinhalte vorangestellt, Ein Anschriftenverzeichnis aller ZDS-Mitgliedsorganisationen beschließt dieses Buch. Die vorliegende Jahresdokumentation der Deutschen Schweineproduktion ist nicht nur für alle Schweinehalter, Zuchtverbănde, -untemehmungen, Lehr- und Forschungsanstalten ein unentbehrliches Arbeitsmaterial, sondern auch fur alle die Schweine- und Tierproduktion tangierenden Entscheidungsträger in der Wirtschaft und Verwaltung.

ERNST RITTER, Dummerstorf

Dieses Buch kann gegen eine Schutzgebuhr von 17,- DM (Ausland 20,- DM) beim ZDS e.V. Adenauerallee 174, S3113-Bonn besiellt werden.

Tel.: 0228/9144740, Fax.: 0228/211777, e-mail: info@ZDS.Bonn.de 